\title{
Exploring Paraconsistency in Degree-Preserving Fuzzy Logics
}

\author{
Rodolfo Ertola ${ }^{1}$ Francesc Esteva ${ }^{2}$ Tommaso Flaminio ${ }^{3}$ Lluís Godo $^{2}$ Carles Noguera ${ }^{4,5}$ \\ ${ }^{1}$ State University of Campinas, Rua Sérgio Buarque de Holanda 251, 13083-859 Campinas, Sao Paulo, Brazil \\ ${ }^{2}$ Artificial Intelligence Research Institute - CSIC, Campus de la UAB s/n, 08193 Bellaterra, Catalonia, Spain \\ ${ }^{3}$ Dipartimento di Scienze Teoriche e Applicate, Università dell'Insubria, Via Mazzini 5, 21100 Varese, Italy \\ ${ }^{4}$ Institute of Information Theory and Automation - AVČR, Pod vodárenskou věží 4, 18208 Prague, Czech Republic \\ ${ }^{5}$ Institute of Computer Science - AVČR, Pod vodárenskou věží 2, 18207 Prague, Czech Republic
}

\begin{abstract}
Paraconsistent logics are specially tailored to deal with inconsistency, while fuzzy logics primarily deal with graded truth and vagueness. In the last decade, mathematical fuzzy logic has been developed as a discipline studying formal many-valued systems arising from fuzzy set theory. In this paper we study to what extent different systems of fuzzy logic are paraconsistent, identifying which families of fuzzy logics satisfy interesting paraconsistency properties.
\end{abstract}

Keywords: Mathematical fuzzy logic, degreepreserving fuzzy logics, paraconsistent logics, logics of formal inconsistency.

\section{Introduction}

Non-classical logics aim to formalize reasoning in a wide variety of different contexts in which the classical approach might be inadequate or not sufficiently flexible. This is typically the case when the information we want to reason about is not perfect, e.g. because it is incomplete, imprecise or contradictory.

On the one hand, fuzzy logics have been proposed as a powerful tool for reasoning with imprecise information, in particular for reasoning with propositions containing vague predicates. Their main feature is that they allow to interpret truth in a linearly ordered scale of truth values which makes them specially suited for representing the gradual aspects of vagueness. Originating from fuzzy set theory [1] they have given rise to the deeply developed area of mathematical fuzzy logic [2] (MFL). Particular deductive systems in MFL have been usually studied under the paradigm of truth-preservation which, generalizing the classical notion of consequence, postulate that a formula follows from a set of premises if every algebraic evaluation that interprets the premises as true also interprets the conclusion as true. Since the notion of truth is interpreted by a designated set of values in the algebras (often just one designated value), it appears that only these truth-values are relevant as regards to consequence. An alternative approach that has recently received some attention is based on the degree-preservation paradigm (see [3, 4]), in which a conclusion follows from a set of premises if for all evaluations its truth degree is not lower than that of the premises. It has been argued that this approach is more coherent with the commitment of manyvalued logics to truth-degree semantics because all values play an equally important rôle in the corresponding notion of consequence (see e.g. [5]).

On the other hand, paraconsistent logics have been introduced, among other approaches (see e.g. [6]), as deductive systems able to cope with contradictions. As much as vagueness, inconsistency is ubiquitious in many contexts in which, regardless of the contradictory information, one is still expected to extract inferences "in a sensible way". Classical logic, and in general any logic validating the ex contradictione quodlibet principle (ECQ), does not allow to reason in any interesting way in the presence of contradictions, since they trivialize deduction and allow to extract any conclusion from an inconsistent theory. They are explosive, in this sense. In contrast, paraconsistent logics are deductive systems where ECQ does not hold, so they allow to tackle with contradictions without trivializing the logic. This kind of systems can be found, for example, in the realm of relevant logics, where their paraconsistency is not their central feature but a by-product of the general principle that one should not infer conclusions which do not bear a "relevant connection" with their premises. Besides those, there have been many studies purposefully focused on paraconsistency giving rise to a variety of logical systems (see e.g. [7]). One such approach are the logics of formal inconsistency (LFIs) studied by the Brazilian school [8]. The main merit of LFIs is that they are paraconsistent logics that manage to internalize the notions of consistency and inconsistency at the object-language level. ${ }^{1}$

Obviously, those phenomena of imperfect information are not mutually independent, but very often found together in many particular examples. Therefore, one might wish for logical systems able

\footnotetext{
${ }^{1}$ It has to be noticed here that in the frame of LFIs the term consistent refers to formulas that basically exhibit a classical logic behaviour, so in particular an explosive behaviour.
} 
to cope with several of them at once. In particular, it would be desirable to have logics for vague and inconsistent information. In this paper we take the first steps towards an approach to this problem which, to the best of our knowledge, has not been considered yet. We want to study, in the context of MFL, paraconsistent fuzzy logics, hoping to have the best of both worlds, i.e. a good tool for reasoning with gradual predicates in possibly contradictory theories. We will argue that the appropriate paradigm for that is not the usual truth-preserving approach, but the degree-preserving one, setting the stage for future development.

After this introduction, Section 2 briefly introduces the necessary basic notions on both paraconsistent and fuzzy logics. Then Section 3 shows that truth-preserving fuzzy logics are explosive, while under some conditions degree-preserving logics are not, and hence they can be seen as paraconsistent systems; we explore their paraconsistent features, give particular examples to illustrate them and characterize a family of LFIs inside fuzzy logics. Finally, since paraconsistency is always defined with respect to a particular negation connective (responsible for the contradictions in inconsistent theories), Section 4 explores alternative negations in fuzzy logics and their interplay with paraconsistency.

\section{Preliminaries}

In this section we introduce the necessary notation and results that will support our investigation. In particular, we briefly present paraconsistent logics and fuzzy logics preserving degrees of truth. We invite the reader to consult [8] and [4] respectively, for more exhaustive treatments of both kinds of logics.

\subsection{About paraconsistency}

As already mentioned above, paraconsistent logics are systems that allow to deal with contradictions without trivializing the logic. In what follows we shall always assume each logic to be finitary, monotonic and to have at least a negation connective that we shall denote, as usual, by $\neg$.

Definition 1. A logic L is explosive (with respect to $\neg$ ) if $\alpha, \neg \alpha \vdash_{\mathrm{L}} \beta$, for every formula $\alpha$ and $\beta$. $\mathrm{L}$ is paraconsistent (with respect to $\neg$ ) if it is not explosive (with respect to $\neg$ ).

Whenever clear from the context, we shall omit to write with respect to which negation a given logic is explosive or paraconsistent. Following [8], paraconsistent logics can be further classified according to several features they exhibit. We provide here the main definitions.

Definition 2. Let $\mathrm{L}$ be a logic and let $\sigma\left(p_{0}, \ldots, p_{n}\right)$ be a formula. L is said to be:

1. partially explosive with respect to $\sigma$ (or $\sigma$ partially explosive), provided that (a) there are formulas $\psi_{0}, \ldots, \psi_{n}$ such that

$$
\forall_{\mathrm{L}} \sigma\left(\psi_{0}, \ldots, \psi_{n}\right) \text {, and }
$$

(b) for all formulas $\psi_{0}, \ldots, \psi_{n}, \varphi$, it holds

$$
\varphi, \neg \varphi \vdash_{\mathrm{L}} \sigma\left(\psi_{0}, \ldots, \psi_{n}\right) .
$$

2. boldly paraconsistent if there is no $\sigma$ such that $\mathrm{L}$ is $\sigma$-partially explosive,

3. controllably explosive in contact with $\sigma\left(p_{0}, \ldots, p_{n}\right)$, if

(a) there are formulas $\alpha, \alpha_{0}, \ldots, \alpha_{n}$ such that $\sigma\left(\alpha_{0}, \ldots, \alpha_{n}\right) \forall_{\mathrm{L}} \alpha$,

(b) there are formulas $\beta, \beta_{0}, \ldots, \beta_{n}$ such that $\neg \sigma\left(\beta_{0}, \ldots, \beta_{n}\right) \nvdash_{\mathrm{L}} \beta$, and

(c) for all formulas $\psi_{0}, \ldots, \psi_{n}, \varphi$, it holds

$$
\sigma\left(\psi_{0}, \ldots, \psi_{n}\right), \neg \sigma\left(\psi_{0}, \ldots, \psi_{n}\right) \vdash_{\mathrm{L}} \varphi .
$$

Johansson's minimal logic [9], where from a contradiction every negation follows, is an example of a logic that is paraconsistent but not boldly paraconsistent.

As a notation, let us write $\bigcirc(p)$ to denote a (possibly empty) set of formulas which only depends on the propositional variable $p$.

Definition 3. Let L be a logic and $\bigcirc(p)$ a set of formulas. L is gently explosive (with respect to $\bigcirc(p))$ if

(a) there are formulas $\varphi_{1}$ and $\psi_{1}$ such that $\bigcirc\left(\varphi_{1}\right), \varphi_{1} \forall_{\mathrm{L}} \psi_{1}$,

(b) there are formulas $\varphi_{2}$ and $\psi_{2}$ such that $\bigcirc\left(\varphi_{2}\right), \neg \varphi_{2} \nvdash_{\mathrm{L}} \psi_{2}$, and

(c) for all formulas $\varphi$ and $\psi$, it holds

$$
\bigcirc(\varphi), \varphi, \neg \varphi \vdash_{\mathrm{L}} \psi \text {. }
$$

If furthermore $\bigcirc(p)$ is finite, we say that $\mathrm{L}$ is finitely gently explosive.

Observe that if $\mathrm{L}$ is finitary and gently explosive, then it is also finitely gently explosive.

Following [8], given a negation $\neg$, we say that a $\operatorname{logic} \mathrm{L}$ is a Logic of Formal Inconsistency (with respect to $\neg),(\neg$-LFI in symbols), if there exists a set of formulas $\bigcirc(p)$ such that $\mathrm{L}$ is $\neg$-gently explosive.

\subsection{About truth-preserving and degree-preserving fuzzy logics}

Truth-preserving fuzzy logics. Most well known and studied system of mathematical fuzzy logic are the so-called t-norm based fuzzy logics, corresponding to formal many-valued calculi with truth-values in the real unit interval $[0,1]$ and with a conjunction and an implication interpreted respectively by a (left-) continuous t-norm and its residuum, and thus, including e.g. the well-known Łukasiewicz and Gödel infinitely-valued logics, corresponding to the calculi defined by Łukasiewicz and min t-norms respectively. The most basic tnorm based fuzzy logic is the logic MTL (monoidal 
t-norm based logic) introduced in [10], whose theorems correspond to the common tautologies of all many-valued calculi defined by a left-continuous tnorm and its residuum [11].

The language of MTL consists of denumerably many propositional variables $p_{1}, p_{2}, \ldots$, binary connectives $\wedge, \&, \rightarrow$, and the truth constant $\overline{0}$. Formulas, which will be denoted by lower case greek letters $\varphi, \psi, \chi, \ldots$, are defined by induction as usual. Further connectives and constants are definable, in particular: $\neg \varphi$ stands for $\varphi \rightarrow \overline{0}$ and $\overline{1}$ stands for $\neg \overline{0}$. A Hilbert-style calculus for MTL was introduced in [10] with the following set of axioms:

(A1) $(\varphi \rightarrow \psi) \rightarrow((\psi \rightarrow \chi) \rightarrow(\varphi \rightarrow \chi))$

(A2) $\varphi \& \psi \rightarrow \varphi$

(A3) $\varphi \& \psi \rightarrow \psi \& \varphi$

(A4) $\varphi \wedge \psi \rightarrow \varphi$

(A5) $\varphi \wedge \psi \rightarrow \psi \wedge \varphi$

(A6) $\varphi \&(\varphi \rightarrow \psi) \rightarrow \varphi \wedge \psi$

(A7a) $(\varphi \rightarrow(\psi \rightarrow \chi)) \rightarrow(\varphi \& \psi \rightarrow \chi)$

$(\mathrm{A} 7 \mathrm{~b})(\varphi \& \psi \rightarrow \chi) \rightarrow(\varphi \rightarrow(\psi \rightarrow \chi))$

$(\mathrm{A} 8)((\varphi \rightarrow \psi) \rightarrow \chi) \rightarrow(((\psi \rightarrow \varphi) \rightarrow \chi) \rightarrow \chi)$

(A9) $\overline{0} \rightarrow \varphi$

and whose unique rule of inference is modus ponens: from $\varphi$ and $\varphi \rightarrow \psi$ derive $\psi$.

MTL is an algebraizable logic in the sense of Blok and Pigozzi [12] and its equivalent algebraic semantics is given by the class of MTL-algebras, that is indeed a variety; call it MTL. MTL-algebras can be equivalently introduced as commutative, bounded, integral residuated lattices $\langle A, \wedge, \vee, \&, \rightarrow, \overline{0}, \overline{1}\rangle$ further satisfying the following prelinearity condition: $(x \rightarrow y) \vee(y \rightarrow x)=\overline{1}$.

Given an MTL-algebra $\boldsymbol{A}=\left\langle A, \wedge^{\boldsymbol{A}}, \vee^{\boldsymbol{A}}, \&^{\boldsymbol{A}}, \rightarrow^{\boldsymbol{A}}\right.$ $\left., \overline{0}^{\boldsymbol{A}}, \overline{1}^{\boldsymbol{A}}\right\rangle$, an $\boldsymbol{A}$-evaluation is any function mapping each propositional variable into $A, e(\overline{0})=\overline{0}^{A}$ and such that, for formulas $\varphi$ and $\psi, e(\varphi \wedge \psi)=e(\varphi) \wedge^{\boldsymbol{A}}$ $e(\psi) ; e(\varphi \vee \psi)=e(\varphi) \vee^{\boldsymbol{A}} e(\psi) ; e(\varphi \& \psi)=e(\varphi) \&^{\boldsymbol{A}}$ $e(\psi) ; e(\varphi \rightarrow \psi)=e(\varphi) \rightarrow^{\boldsymbol{A}} e(\psi)$. An evaluation $e$ is said to be a model for a set of formulas $\Gamma$, if $e(\gamma)=\overline{1}^{\boldsymbol{A}}$ for each $\gamma \in \Gamma$.

We shall henceforth adopt a lighter notation dropping the superscript $\boldsymbol{A}$. The distinction between a syntactic object and its interpretation in an algebraic structure will be always clear by the context.

The algebraizability gives the following strong completeness theorem:

For every set $\Gamma \cup\{\varphi\}$ of formulae, $\Gamma \vdash_{\mathrm{MTL}} \varphi$ iff for every $\boldsymbol{A} \in \mathbb{M T L}$ and every $\boldsymbol{A}$-evaluation $e$, if $e$ is a model of $\Gamma$ then $e$ is a model of $\varphi$ as well.

For this reason, since the consequence relation amounts to preservation of the truth-constant $\overline{1}$, MTL can be called a truth-preserving logic.

Actually, the algebraizability is preserved for any logic L that is an axiomatic expansion of MTL satisfying the following congruence property
(Cng) $\varphi \rightarrow \psi, \psi \rightarrow \varphi \vdash_{\mathrm{L}} c\left(\chi_{1}, \ldots, \chi_{i}, \varphi, \ldots, \chi_{n}\right)$ $\rightarrow c\left(\chi_{1}, \ldots, \chi_{i}, \psi, \ldots, \chi_{n}\right)$

for any possible new $n$-ary connective $c$ and each $i<n$. This is due to the fact that such axiomatic expansions (also called core fuzzy logics) are in fact a Rasiowa-implicative logics (cf. [13]), and as proved in [14], every Rasiowa-implicative logic L is algebraizable and, if it is finitary, its equivalent algebraic semantics, the class $\mathbb{L}$ of L-algebras, is a quasivariety (a variety in the case of a core fuzzy logic).

As a consequence, any core fuzzy logic L enjoys the same kind of completeness theorem with respect to the corresponding L-algebras. But more than that, the variety of L-algebras can also be shown to be generated by the subclass of all its linearly ordered members [14]. ${ }^{2}$ This means that any core fuzzy logic $\mathrm{L}$ is strongly complete with respect to the class of L-chains, that is, core fuzzy logics are semilinear.

Actually, one can also expand MTL with the Monteiro-Baaz projection connective $\triangle$, obtaining again a finitary Rasiowa-implicative semilinear logic MTL $_{\triangle}$. Then, one analogously defines $\triangle$-core fuzzy logics as axiomatic expansions of $\mathrm{MTL}_{\triangle}$ satisfying (Cng) for any possible new connective.

Semilinearity can also be inherited by many expansions of $(\triangle$-)core fuzzy logics with new (finitary) inference rules. Indeed, in [14] it is shown that an expansion L of a core fuzzy logic is semilinear iff for each newly added finitary inference rule

(R) from $\Gamma$ derive $\varphi$,

its corresponding $\vee$-form

$\left(\mathbf{R}^{\vee}\right)$ from $\Gamma \vee p$ derive $\varphi \vee p$

is derivable in $\mathrm{L}$ as well, where $p$ is an arbitrary propositional variable not appearing in $\Gamma \cup\{\varphi\}$.

Degree-preserving fuzzy logics. It is clear that $(\triangle$-) core fuzzy logics, like MTL, are truthpreserving fuzzy logics. But besides the truthpreserving paradigm that we have so far considered, one can find an alternative approach in the literature. Given a $(\triangle$-)core fuzzy logic $\mathrm{L}$, and based on the definitions in [4], we can introduce a variant of $\mathrm{L}$ that we shall denote by $\mathrm{L} \leq$, whose associated deducibility relation has the following semantics: for every set of formulas $\Gamma \cup\{\varphi\}, \Gamma \vdash_{\mathrm{L}} \leq \varphi$ iff for every L-chain $\boldsymbol{A}$, every $a \in A$, and every $\boldsymbol{A}$-evaluation $v$, if $a \leq v(\psi)$ for every $\psi \in \Gamma$, then $a \leq v(\varphi)$. For this reason $\mathrm{L} \leq$ is known as a fuzzy logic preserving degrees of truth, or the degree-preserving companion of $\mathrm{L}$. In this paper, we often use generic

\footnotetext{
${ }^{2}$ Moreover, for a number of core fuzzy logics, including MTL, it has been shown that their corresponding varieties are also generated by the subclass of MTL-chains defined on the real unit interval, called standard algebras. For instance, MTL is also complete wrt standard MTL-chains, that are of the form $[0,1]_{*}=\left\langle[0,1], \min , \max , *, \rightarrow_{*}, 1,0\right\rangle$ of type $\langle 2,2,2,2,0,0\rangle$, where $*$ denotes a left-continuous t-norm and $\rightarrow_{*}$ is its residuum [11].
} 
statements about "every logic $\mathrm{L} \leq$ " referring to "the degree-preserving companion of any $(\triangle$-)core fuzzy logic L".

As regards to axiomatization, if $\mathrm{L}$ is a core fuzzy logic, then the logic $\mathrm{L} \leq$ admits a Hilbert-style axiomatization having the same axioms as $\mathrm{L}$ and the following deduction rules [4]:

$(\mathbf{A d j}-\wedge)$ from $\varphi$ and $\psi$ deduce $\varphi \wedge \psi$

(MP-r) if $\vdash_{\mathrm{L}} \varphi \rightarrow \psi$ (i.e. if $\varphi \rightarrow \psi$ is a theorem of $\mathrm{L})$, then from $\varphi$ and $\varphi \rightarrow \psi$ derive $\psi$

Note that if the set of theorems of $\mathrm{L}$ is decidable, then the above is in fact a recursive Hilbert-style axiomatization of $\mathrm{L} \leq$.

In general, let L be a Rasiowa-implicative expansion of MTL with a set of new inference rules

$\left(\mathbf{R}_{i}\right)$ from $\Gamma_{i}$ derive $\varphi_{i}$,

for $i \in I$. Then we have the following result.

Proposition 4. $\mathrm{L} \leq$ is axiomatized by adding to the axioms of $\mathrm{L}$ the above two inference rules plus the following restricted rules

$\left(\mathbf{R}_{i}-r\right)$ If $\vdash_{\mathrm{L}} \Gamma_{i}$, then from $\Gamma_{i}$ derive $\varphi_{i}$

for each $i \in I$.

In particular, if $\mathrm{L}$ is a $\triangle$-core fuzzy logic, then the only rule one should add is the following restricted necessitation rule for $\triangle$ :

$(\triangle-r)$ if $\vdash_{\mathrm{L}} \varphi$, then from $\varphi$ derive $\triangle \varphi$

The following proposition points out some key analogies and differences between $\mathrm{L}$ and $\mathrm{L} \leq$ that we shall use in the rest of this paper.

\section{Proposition 5. The following facts hold:}

(1) The two logics $\mathrm{L}$ and $\mathrm{L} \leq$ have the same theorems: $\vdash_{\mathrm{L}} \varphi$ iff $\vdash_{\mathrm{L}} \leq \varphi$.

(2) For all formulas $\varphi, \psi$ one has:

(i) $\varphi, \psi \vdash_{\mathrm{L}} \varphi \& \psi ; \quad \varphi, \psi \vdash_{\mathrm{L}} \varphi \wedge \psi$;

(ii) $\varphi, \psi \vdash_{\mathrm{L} \leq} \varphi \wedge \psi$.

(3) $\varphi_{1}, \ldots, \varphi_{n} \vdash_{\mathrm{L} \leq} \psi$ iff $\vdash_{\mathrm{L}}\left(\varphi_{1} \wedge \ldots \wedge \varphi_{n}\right) \rightarrow \psi$.

Last item (3) interestingly points out that, indeed, deductions from a finite set of premises in $\mathrm{L} \leq$ exactly correspond to theorems in L.

\section{Paraconsistent fuzzy logics}

The first important observation is that $(\triangle$-)core fuzzy logics as studied in the truth-preservation paradigm do not have any paraconsistent feature regarding their residual negation $\neg$.

Proposition 6. ( $\triangle$-) Core fuzzy logics are explosive with respect to $\neg$.

Proof. The proof is easy because in these logics it holds: $\varphi, \neg \varphi \vdash \varphi \& \neg \varphi$, and $\varphi \& \neg \varphi \vdash \overline{0}$.
Thus, $(\triangle$-)core fuzzy logics are not paraconsistent. In contrast, their degree-preserving companions are paraconsistent provided that they do not satisfy the pseudo-complementation law.

Proposition 7. Let L be a ( $\triangle$-) core fuzzy logic. Then $\mathrm{L} \leq$ is paraconsistent iff $\mathrm{L}$ is not pseudocomplemented.

Proof. By definition, a logic is pseudocomplemented iff it has $(\varphi \wedge \neg \varphi) \rightarrow \overline{0}$ as theorem. So we have: $\vdash_{\mathrm{L} \leq}(\varphi \wedge \neg \varphi) \rightarrow \overline{0}$ iff $\vdash_{\mathrm{L}}(\varphi \wedge \neg \varphi) \rightarrow \overline{0}$ (both logics have the same theorems) iff $\mathrm{L}$ is pseudo-complemented. Now, if $\vdash_{\mathrm{L} \leq}(\varphi \wedge \neg \varphi) \rightarrow \overline{0}$, then $\mathrm{L} \leq$ is explosive because $\varphi, \neg \varphi \vdash_{\mathrm{L}} \leq \varphi \wedge \neg \varphi$ and $\vdash_{\mathrm{L} \leq} \overline{0} \rightarrow \psi$. Conversely, if $\nvdash_{\mathrm{L}} \leq(\varphi \wedge \neg \varphi) \rightarrow \overline{0}$, then there exists an L-chain $\boldsymbol{A}$ and $a \in A$ such that $a \wedge \neg a>\overline{0}$ and therefore we also have an example showing that $p, \neg p \forall_{\mathrm{L} \leq} q$ (taking $e(p)=a$ and $e(q)=\overline{0})$, and thus the logic is paraconsistent.

Next, we study what kinds of paraconsistency properties those logics enjoy. The first obvious question is whether they are boldly paraconsistent or partially explosive with respect to some formula.

Proposition 8. Every logic $\mathrm{L} \leq$ is partially explosive with respect to $\sigma(p)=p \vee \neg p$.

Proof. L proves Kleene's axiom $(\varphi \wedge \neg \varphi) \rightarrow(\psi \vee \neg \psi)$ because it is complete with respect to the chains of the corresponding variety. Therefore, we have $\varphi, \neg \varphi \vdash_{\mathrm{L}} \leq \psi \vee \neg \psi$. On the other hand, if $\mathrm{L}$ is not classical logic, $\psi \vee \neg \psi$ is not a theorem of $\mathrm{L} \leq$ (if $\mathrm{L}$ is classical logic then so is $\mathrm{L} \leq$, and hence it is explosive).

Therefore, logics $\mathrm{L} \leq$ may be paraconsistent, but they are never boldly paraconsistent. When it comes to controllable explosion, we can characterize the class of such logics which are controllably explosive in terms of the following notion of locally Boolean logic.

Definition 9. A logic $\mathrm{L} \leq$ is locally Boolean if there exists a formula $\sigma$ such that $\forall_{\mathrm{L} \leq} \neg \sigma, \forall_{\mathrm{L}} \leq \neg \neg \sigma$, and for every L-chain $\boldsymbol{A}$ and every $\boldsymbol{A}$-evaluation $v, v(\neg \sigma) \in\{\overline{0}, \overline{1}\}$.

Proposition 10. A logic $\mathrm{L} \leq$ is controllably explosive iff it is locally Boolean.

Proof. Assume that $\mathrm{L} \leq$ is controllably explosive w.r.t. a formula $\sigma\left(p_{0}, \ldots, p_{n}\right)$. This means that there are $\alpha, \alpha_{0}, \ldots, \alpha_{n}$ such that $\sigma\left(\alpha_{0}, \ldots, \alpha_{n}\right) \nvdash_{\mathrm{L}} \leq \alpha$, and there are $\beta, \beta_{0}, \ldots, \beta_{n}$ such that $\neg \sigma\left(\beta_{0}, \ldots, \beta_{n}\right) \forall_{\mathrm{L}} \leq \beta$; moreover, for every $\gamma_{0}, \ldots, \gamma_{n}, \gamma$, it holds

$$
\sigma\left(\gamma_{0}, \ldots, \gamma_{n}\right), \neg \sigma\left(\gamma_{0}, \ldots, \gamma_{n}\right) \vdash_{\mathrm{L} \leq} \gamma .
$$

Therefore, by the chain-completeness theorem, the above holds iff for every L-chain $\boldsymbol{A}$, and every $\boldsymbol{A}$ evaluation $v$

$$
v\left(\sigma\left(\gamma_{0}, \ldots, \gamma_{n}\right) \wedge \neg \sigma\left(\gamma_{0}, \ldots, \gamma_{n}\right)\right)=\overline{0} .
$$


Then either $v\left(\sigma\left(\gamma_{0}, \ldots, \gamma_{n}\right)\right)=\overline{0}, \quad$ and hence $v\left(\neg \sigma\left(\gamma_{0}, \ldots, \gamma_{n}\right)\right)=\overline{1}$, or otherwise $v\left(\neg \sigma\left(\gamma_{0}, \ldots, \gamma_{n}\right)\right)=\overline{0} . \quad$ Moreover, from the existence of $\alpha, \alpha_{0}, \ldots, \alpha_{n}$ such that $\sigma\left(\alpha_{0}, \ldots, \alpha_{n}\right) \quad \forall_{\mathrm{L}} \leq \alpha$, we infer that there must exist an L-chain $\boldsymbol{B}$ and a $\boldsymbol{B}$-evaluation $e$ such that $e(\sigma) \neq \overline{0}$, and hence $\nvdash_{\mathrm{L}} \leq \neg \sigma$. Similarly, from $\neg \sigma\left(\beta_{0}, \ldots, \beta_{n}\right) \forall_{\mathrm{L}} \leq \beta$, we know that there is an L-chain $\boldsymbol{C}$ and a $\boldsymbol{C}$-evaluation $e^{\prime}$ such that $e^{\prime}(\neg \sigma) \neq \overline{0}$; therefore we have $e^{\prime}(\sigma)=\overline{0}$ and thus $e^{\prime}(\neg \neg \sigma)=\overline{0}$ and $\nvdash_{\mathrm{L}} \leq \neg \neg \sigma$. Therefore $\mathrm{L} \leq$ is locally Boolean.

Now assume that $\mathrm{L} \leq$ is locally Boolean, i.e. there is $\sigma$ such that $\forall_{\mathrm{L}} \leq \neg \sigma, \forall_{\mathrm{L}} \leq \neg \neg \sigma$, and for every L-chain $\boldsymbol{A}$ and every $\boldsymbol{A}$-evaluation $v, v(\neg \sigma) \in$ $\{\overline{0}, \overline{1}\}$. Let $p_{0}, \ldots, p_{n}$ be the variables occurring in $\sigma$. Thus, for every substitution of $p_{0}, \ldots, p_{n}$ by arbitrary formulas $\gamma_{0}, \ldots, \gamma_{n}, v\left(\neg \sigma\left(\gamma_{0}, \ldots, \gamma_{n}\right)\right) \in$ $\{\overline{0}, \overline{1}\}$. Thus, either $v\left(\neg \sigma\left(\gamma_{0}, \ldots, \gamma_{n}\right)\right)=\overline{0}$, or $v\left(\neg \sigma\left(\gamma_{0}, \ldots, \gamma_{n}\right)\right)=\overline{1}$ and hence $v\left(\sigma\left(\gamma_{0}, \ldots, \gamma_{n}\right)\right)=$ $\overline{0}$. Therefore, for every $\gamma_{0}, \ldots, \gamma_{n}, \gamma$,

$$
\overline{0}=v\left(\sigma\left(\gamma_{0}, \ldots, \gamma_{n}\right) \wedge \neg \sigma\left(\gamma_{0}, \ldots, \gamma_{n}\right)\right) \leq v(\gamma),
$$

that is,

$$
\sigma\left(\gamma_{0}, \ldots, \gamma_{n}\right), \neg \sigma\left(\gamma_{0}, \ldots, \gamma_{n}\right) \vdash_{\mathrm{L} \leq} \gamma .
$$

On the other hand, since $\nvdash_{\mathrm{L} \leq} \neg \sigma$, there is an Lchain $\boldsymbol{B}$ and a $\boldsymbol{B}$-evaluation $e$ such that $e(\neg \sigma) \neq \overline{1}$ and hence $e(\sigma) \neq \overline{0}$. Similarly, since $\nvdash_{\mathrm{L}} \leq \neg \neg \sigma$, there is an L-chain $\boldsymbol{C}$ and a $\boldsymbol{C}$-evaluation $e^{\prime}$ such that $e(\neg \neg \sigma) \neq \overline{1}$ and hence $e(\neg \sigma) \neq \overline{0}$. Hence L is controllably explosive.

We explore the property of locally Boolean logics and show its extent in the following examples.

Example 1. Let $\boldsymbol{C}$ be an MTL-chain such that the Monteiro-Baaz operator $\triangle$ is definable by a term $\delta(p)$ in all chains of the variety generated by $\boldsymbol{C}$. Then both the degree-preserving logic of $\boldsymbol{C}$ and that logic of any ordinal sum $\boldsymbol{C} \oplus \boldsymbol{A}$ where $\boldsymbol{A}$ is any MTL-chain are locally Boolean with $\sigma(p)=\neg \delta(p)$. The result is obvious for the logic of $\boldsymbol{C}$. In the other case, observe that for any evaluation $v$ on $\boldsymbol{C} \oplus \boldsymbol{A}$, $v(\sigma(p))=\overline{1}$ if $v(p) \in C$ and $v(\sigma(p))=\overline{0}$ if $v(p) \in$ $A$. Observe that all finite $\mathrm{MV}$-chains fall under the scope of this example.

Example 2. Let $\boldsymbol{C}$ be an MTL-chain. The set of its positive and negative elements are respectively defined as $C_{+}=\{a \in A \mid a>\neg a\}$ and $C_{-}=\{a \in$ $A \mid a \leq \neg a\}$. Assume that $C_{+}$is an MTL-filter, i.e. non-empty, upset w.r.t. the order and closed under \&. This means that $C_{+}$coincides with the radical of $\boldsymbol{C}$ (see e.g. [15]). In this case, the quotient algebra $\boldsymbol{C} / C_{+}$is the two-element Boolean algebra $\boldsymbol{B}_{2}$, if $\boldsymbol{C}$ has no negation fixpoint, or the three-element MValgebra $\mathbf{\mathbf { L }}_{3}$ otherwise. In both cases the logic of $\boldsymbol{C}$ is locally Boolean with the formula $\sigma(p)=\left(\neg\left(p^{2}\right)\right)^{2} .^{3}$

\footnotetext{
${ }^{3}$ In general, $\varphi^{n}$ is an abbreviation for $\varphi \& .{ }^{n} . \& \varphi$.
}

Indeed, it is easy to see that $\sigma(x)=\overline{1}$ if $x \in C_{-}$ and $\sigma(x)=\overline{0}$ if $x \in C_{+}$. Examples of MTL-chains satisfying this condition are the Chang MV-algebra, and any WNM-chain (thus including NM-chains).

Example 3. Let $\boldsymbol{C}$ be the standard MV-chain $[0,1]_{E}$ or any ordinal sum beginning with a copy of $[0,1]_{E}$. Then the logic of $\boldsymbol{C}$ is not locally Boolean. The first case is obvious because any function associated to any formula has to be piecewise linear and continuous (a McNaughton function [16] in particular) while the function associated to the required formula $\sigma$ for the logic to be locally Boolean must be discontinuous at 0 and at 1 . For the ordinal sum case, suppose that $\sigma\left(p_{1}, p_{2}, \ldots, p_{k}\right)$ is a formula satisfying the required condition. Take the restriction of this formula to evaluations taking values on the first component of the ordinal sum. This evaluation has to define a McNaughton function but at it also has to be discontinuous in 0 , which is impossible.

Finally, let us consider the notion of gently explosive logic with respect to a set of formulas $\bigcirc(p)$. Recall Definition 11 and assume that $\mathrm{L}$ is a $(\triangle$-)core fuzzy logic complete with respect to a single L-chain $\boldsymbol{A}$. Then, thanks to the fact that $\mathrm{L} \leq$ is finitary and the presence of the adjunction rule $(\mathbf{A d j}-\Lambda)$, we can assume that $\bigcirc(p)$ is just one formula and the definition can be reformulated in semantical terms as follows:

(a) there are formulas $\varphi_{1}, \psi_{1}$ and an $\boldsymbol{A}$-evaluation $e_{1}$ such that $e_{1}\left(\bigcirc\left(\varphi_{1}\right) \wedge \varphi_{1}\right)>e_{1}\left(\psi_{1}\right)$,

(b) there are formulas $\varphi_{2}, \psi_{2}$ and an $\boldsymbol{A}$-evaluation $e_{2}$ such that $e_{2}\left(\bigcirc\left(\varphi_{2}\right) \wedge \neg \varphi_{2}\right)>e_{2}\left(\psi_{2}\right)$,

(c) for every formulas $\varphi, \psi$ and each $\boldsymbol{A}$-evaluation $e, e(\bigcirc(\varphi) \wedge \varphi \wedge \neg \varphi) \leq e(\psi)$.

Proposition 11. Let $\mathrm{L} \leq$ be the logic of a chain $\boldsymbol{A}$. Then the following are equivalent:

1. $\mathrm{L} \leq$ is gently explosive;

2. There exists a term $\bigcirc(p)$ such that

- $\bigcirc(\overline{0})>\overline{0}$,

- there is $x \in A$ with $\neg x=\overline{0}$ and $\bigcirc(x)>\overline{0}$,

- $\bigcirc(t)=\overline{0}$ for each $t \in A$ such that $t, \neg t>\overline{0}$.

Proof. Assume that $\mathrm{L} \leq$ is gently explosive. Then there exists a formula $\bigcirc(p)$ satisfying the reformulation mentioned above. Thus there are $x, y \in A$, such that $x \wedge \bigcirc(x)>\overline{0}$ and $\bigcirc(y) \wedge \neg y>\overline{0}$, and for every $z \in A z \wedge \neg z \wedge \bigcirc(z)=\overline{0}$. It is clear that the equality for every $z \in A$ implies the last condition from 2. From the properties of $x$ and $y$ it follows that $x>\overline{0}, \neg x=\overline{0}$, and $y=\overline{0}$, and hence $\bigcirc(\overline{0})>\overline{0}$, so the remaining two conditions are satisfied. The converse direction is also very easy to check.

We have, therefore, identified the conditions for the degree-preserving fuzzy logic of an (expansion of an) MTL-chain to be an $\neg$-LFI. 
Example 4. The degree-preserving logic of the standard MV-chain $[0,1]_{E}$ is not gently explosive. In fact, as we recalled in Example 3, every definable term of such logic corresponds to a McNaughton function [16], and McNaughton functions, being continuous functions, they cannot satisfy the conditions of the previous theorem.

Example 5. If L has the Monteiro-Baaz's $\triangle$ connective (as primitive or definable), then $\mathrm{L} \leq i$ sently explosive with $\bigcirc(\alpha)=\triangle(\alpha \vee \neg \alpha)$, as one can easily check from the conditions of the previous proposition. This is the case of the logic of a finite MVchain $E_{n}\left(\triangle(\varphi)=\varphi^{n}\right)$ or, more in general, the logic of an $\mathrm{S}_{n} \mathrm{MTL}-$ chain $\left(\triangle(\varphi)=\neg \varphi^{n} \vee \varphi\right)$ [17].

\section{Expansion of fuzzy logics by further negations}

Let $\mathrm{L}$ be an axiomatic extension of MTL. Next subsections are devoted to the study of expansions of $\mathrm{L}$ and $\mathrm{L} \leq$ obtained by adding either the dual intuitionistic negation $D$, or an involutive negation $\sim$. In what follows we will denote by $\mathrm{L}_{D}$ and $\mathrm{L}_{D}^{\leq}$, and by $\mathrm{L}_{\sim}$ and $\mathrm{L} \stackrel{\leq}{\sim}$, the expansions of $\mathrm{L}$ and $\mathrm{L} \leq$ with $D$ and $\sim$ respectively.

\subsection{Adding dual intuitionistic negation}

Dual intuitionistic negation was introduced by Cecylia Rauszer in [18], in the framework of what she called semi-Boolean algebras, expansions of Heyting algebras with the dual operator $\rightarrow$ of the relative pseudo complement $\rightarrow$. This dual operator, called pseudo-difference, is required to satisfy the following condition: $a \rightarrow b \leq c$ iff $a \leq b \vee c$. Dual intuitionistic negation, that is called $\vee$-complement in [18], and here denoted $D$, is the negation associated to $\rightarrow$, i.e. $D(x)=1 \rightarrow x$. This negation is after used by Priest [19] in the setting of paraconsistent systems and also studied by Ertola et al. in the context of Intuitionism in [20], and in [21] in the context of the logics of formal inconsistency, studied by Carnielli et al. in [8]. In this section we will study $D$-paraconsistency in the setting of fuzzy logics. First we introduce a Hilbert-style axiomatization of truth-preserving fuzzy logics $\mathrm{L}_{D}$, and after we study their degree-preserving counterparts $\mathrm{L}_{D}^{\leq}$. Among other things, we show that logics $\mathrm{L}_{D}^{\leq}$are $D$ paraconsistent and study some of their properties.

We formally define $\mathrm{L}_{D}$ as the logic obtained by expanding the language of L by the unary connective $D$ and whose additional axioms and rule are as follows:
(D1) $\varphi \vee D \varphi$
(D2) $D \varphi \vee \neg D \varphi$
(DR) from $\varphi \vee \psi$ derive $D \varphi \rightarrow \psi$

Indeed, this system corresponds to the strengthening with (D2) of the axiomatization of the dual of intuitionistic negation $D$, in the frame of intuitionistic logic, presented in [20] and consisting of (D1) and (DR). Actually, it can be checked that an equivalent and simpler axiomatization of $\mathrm{L}_{D}$ is the following one, that we will use from now on:

(D1) $\varphi \vee D \varphi$

(DN) from $\varphi \vee \psi$ derive $\neg D \varphi \vee \psi$

First of all, we show that $\mathrm{L}_{D}$ satisfies the congruence property (Cng) for $D$.

Lemma 12. In $\mathrm{L}_{D}$ the following deduction holds:

$$
\varphi \rightarrow \psi \vdash_{\mathrm{L}_{D}} D \psi \rightarrow D \varphi .
$$

Proof. First of all, applying the rule (DN) to axiom (D1) one proves that $D \varphi \vee \neg D \varphi$ is a theorem of $\mathrm{L}_{D}$. Now, in MTL the following deduction holds: $\psi \vee \neg \psi \vdash_{\text {MTL }}(\psi \rightarrow \varphi) \leftrightarrow(\neg \psi \vee \varphi)$. Therefore, in $\mathrm{L}_{D},(D \psi \rightarrow D \varphi) \leftrightarrow(\neg D \psi \vee D \varphi)$ is also a theorem. Finally, from $\varphi \rightarrow \psi$ and $\varphi \vee D \varphi$ one can easily prove $\psi \vee D \varphi$, and using (DN) one obtains $\neg D \psi \vee D \varphi$, and hence, $D \psi \rightarrow D \varphi$ as well.

Therefore, it follows that the congruence condition (Cng) for $D$ indeed holds, and thus $\mathrm{L}_{D}$ is a Rasiowa-implicative logic. Since the rule (DN) is closed under $\vee$-forms, it follows that $\mathrm{L}_{D}$ is semilinear as well. The corresponding algebraic semantics is given by the class of $\mathrm{L}_{D}$-algebras, structures $\langle A, \wedge, \vee, \&, \rightarrow, D, \overline{0}, \overline{1}\rangle$ such that the $D$-free reduct is an L-algebra and the two following properties hold for each $x \in A$ :

- $x \vee D(x)=\overline{1}$;

- if $x \vee y=\overline{1}$ then $\neg D(x) \vee y=\overline{1}$.

From this definition, the class of $\mathrm{L}_{D}$-algebras is a quasivariety, but later we shall see that it is indeed a variety. Since $\mathrm{L}_{D}$ is semilinear, it is complete with respect to the class of $\mathrm{L}_{D}$-chains. Moreover, it is easy to check that if $\mathrm{L}$ is standard complete, then so it is $\mathrm{L}_{D}$. Notice that in an $\mathrm{L}_{D}$-chain, the two conditions above univocally force the $D$ operator to be of the following form:

$$
D(x)= \begin{cases}\overline{1}, & \text { if } x<\overline{1} \\ \overline{0}, & \text { if } x=\overline{1}\end{cases}
$$

Therefore, $D$ is the dual intuitionistic negation (it satisfies $D(x)=\min \{y \mid x \vee y=1\}$ ), and also $\neg D$ is exactly the Monteiro-Baaz $\triangle$ operator in a chain. Indeed one can check that the logic $\mathrm{L}_{D}$ is equivalent to $\mathrm{L}_{\triangle}$, since in $\mathrm{L}_{\triangle}$ the connective $D$ is definable as

$$
D(\varphi):=\neg \triangle(\varphi)
$$

and, vice-versa, in $\mathrm{L}_{D}$ the $\triangle$ is definable as

$$
\triangle(\varphi):=\neg D(\varphi) .
$$

Thus, in fact, $\mathrm{L}_{D}$ can be considered as a $\triangle$-core fuzzy logic. 
Proposition 13. The logic $\mathrm{L}_{D}$ is not Dparaconsistent.

Proof. It is enough to observe that there is no $\mathrm{L}_{D^{-}}$ chain $\boldsymbol{A}$ such that $e(\varphi)=e(D \varphi)=1$ for some $\mathbf{A}$ evaluation $e$, since $e(D \varphi)=1$ iff $e(\varphi)<1$.

The logic $\mathrm{L}_{\bar{D}}$ is defined from $\mathrm{L}_{D}$ as usual, that is, with the same axioms but restricting the rule (DN) to apply only to theorems, in other words, by replacing $(\mathrm{DN})$ by the following rule:

(DN-r) If $\vdash_{\mathrm{L}_{D}} \varphi \vee \psi$, from $\varphi \vee \psi$ derive $\neg D \varphi \vee \psi$

It is very easy to check that for any $\mathrm{L}, \mathrm{L}-\frac{\leq}{D}$ is $D$-paraconsistent. Indeed, it is clear that in any $\mathrm{L}_{D}$-chain, $x \wedge D(x)>0$ for $0<x<1$. Hence, for instance it holds that $\varphi, D \varphi \nvdash_{\mathrm{L}} \leq \overline{0}$. It is also clear that, if $p$ and $q$ are two different propositional variables, then $p, D p \nvdash_{\mathrm{L}_{\bar{D}}} q$. Moreover, we can show that the logics $\mathrm{L} \leq$ are indeed both boldly and gently $D$-paraconsistent.

Proposition 14. For any $\mathrm{L}$, the logic $\mathrm{L}_{\bar{D}}^{\leq}$is boldly and gently D-paraconsistent.

Proof. Suppose that $\psi=\sigma\left(p_{1}, \ldots, p_{n}\right)$ such that $\nvdash_{\mathrm{L}}$ $\psi$. Then there exists an evaluation $v$ on some $\mathrm{L}_{D^{-}}$ chain such that $v(\psi)<1$. In order to prove that $\mathrm{L}_{D}$ is boldly $D$-paraconsistent is enough to show that there exists a formula $\varphi$ such that $\varphi, D \varphi \nvdash_{\mathrm{L}_{\bar{D}}} \psi$. Let $\varphi=q$ be a variable not occurring in $\psi$. Then, define a $\mathrm{L}_{D}$-evaluation $v^{\prime}$ such that $v^{\prime}\left(p_{i}\right)=v\left(p_{i}\right)$ for each $i=1, \ldots, n$ and $v^{\prime}(q)=\beta$ such that $1>\beta>$ $v^{\prime}(\psi)=v(\psi)$. Then we clearly have $v^{\prime}(q \wedge D q)=$ $v^{\prime}(q)>v^{\prime}(\psi)$, and hence $p, D p \nvdash_{\mathrm{L}_{D}} \psi$, and hence $\mathrm{L}_{\bar{D}}^{\leq}$is not partially explosive with respect to any $\sigma$.

In order to prove that $\mathrm{L}_{D}$ is gently $D$ paraconsistent, take $\bigcirc(p)=\triangle(p \vee \neg p)=\neg D(p \vee$ $\neg p)$. An easy computation shows that the formula $\bigcirc(p)$ satisfies the required conditions.

Notice that the usual argument using Kleene axioms showing that a degree-preserving fuzzy logic $\mathrm{L} \leq$ is partially explosive (and hence not boldly paraconsistent) used in Proposition 8 cannot be applied when the considered negation is the dual intuitionistic negation D. For instance $\mathrm{L}_{\bar{D}}$ is not partially $D$-explosive although Kleene axiom $(\varphi \wedge D \varphi) \rightarrow$ $(\psi \vee D \psi)$ trivially holds since the formula $\psi \vee D \psi$ is a theorem of $\mathrm{L} \frac{\leq}{D}$.

\subsection{Adding an involutive negation}

As far as we know, expansions with an involutive negation have only been studied when considered together with the Monteiro-Baaz $\triangle$ operator [22]. Here we define an expansion of a core fuzzy logic $\mathrm{L}$ by an involutive negation without using $\triangle{ }^{4}$ We

\footnotetext{
${ }^{4}$ Of course, the interesting case is when the negation $\neg$ of $\mathrm{L}$ is not involutive.
}

define the $\operatorname{logic} \mathrm{L}_{\sim}$ as the expansion of $\mathrm{L}$ by a new unary connective $\sim$ with the following additional axiom and rule:

$(\sim) \sim \sim \varphi \leftrightarrow \varphi$

(OR) from $(\varphi \rightarrow \psi) \vee \chi$ derive $(\sim \psi \rightarrow \sim \varphi) \vee \chi$

Note that, using $(\sim)$ and $(\mathrm{OR})$, one can show that $\sim \overline{1} \leftrightarrow \overline{0}$ and $\sim \overline{0} \leftrightarrow \overline{1}$.

An $\mathrm{L}_{\sim}$-algebra is a structure $\langle A, \wedge, \vee, \&, \rightarrow$ $, \sim, \overline{0}, \overline{1}\rangle$ such that the $\sim$-free reduct is an L-algebra and the two following properties hold for each $x, y, z \in A$ :

$$
\begin{aligned}
& \text { - } \sim \sim x=x \\
& \text { - if }(x \rightarrow y) \vee z=1 \text { then }(\sim y \rightarrow \sim x) \vee z=1 .
\end{aligned}
$$

From [14] it is immediate that $\mathrm{L}_{\sim}$ is complete w.r.t. the class of all $\mathrm{L}_{\sim}$-algebras and more specifically w.r.t. the class of $\mathrm{L}_{\sim}$-chains.

Given the axiomatization of $\mathrm{L}_{\sim}$, we can easily obtain an axiomatization of $\mathrm{L} \lesssim$ just by replacing the $(\mathrm{OR})$ rule by its restriction to theorems:

(OR-r) if $\vdash_{\mathrm{L}_{\sim}}(\varphi \rightarrow \psi) \vee \chi$ from $(\varphi \rightarrow \psi) \vee \chi$ derive $(\sim \psi \rightarrow \sim \varphi) \vee \chi$

Now we turn our attention to paraconsistency with respect to $\sim$.

Proposition 15. $\mathrm{L}_{\sim}$ is not $\sim$-paraconsistent but $\mathrm{L} \lesssim$ is always $\sim$-paraconsistent.

Proof. Observe that there is no evaluation $e$ such that $e(\varphi)=e(\sim \varphi)=1$, and hence, for each formula $\varphi,\{\varphi, \sim \varphi\} \vdash_{\mathrm{L}_{\sim}} \psi$ for any $\psi$, and thus $\mathrm{L}_{\sim}$ is explosive. On the other hand, the proof that the logic $\mathrm{L} \lesssim$ is $\sim$-paraconsistent is the same as in Proposition 7 , the main difference being that the logic $\mathrm{L} \lesssim$ is $\sim$-paraconsistent for any axiomatic extension of MTL, and not only for non SMTL extensions, because $\sim$ is involutive. Indeed, if $\boldsymbol{A}$ is an $\mathrm{L}_{\sim}$-chain with more than two elements, one can always find an $\boldsymbol{A}$-evaluation $e$ with $e(p \wedge \sim p)>0$.

Proposition 16. $\mathrm{L} \lesssim$ is partially $\sim$-explosive with respect to $\sigma(p)=p \vee \sim p$.

Proof. It is obvious that Kleene's axiom is also valid for the negation $\sim$. Then the proof of Proposition 8 is also valid and therefore the logic $\mathrm{L} \lesssim$ is partially $\sim$-explosive.

Finally, whether $\mathrm{L} \lesssim$ is $\sim$-gently paraconsistent depends on the initial logic L. For example, if L is an axiomatic extension of SMTL, then in the $\mathrm{L}_{\sim}$ the operator $\triangle$ is definable as the composition of the two negations $\neg$ and $\sim$, and thus $\mathrm{L}_{\sim}$ is gently $\sim$ paraconsistent. Indeed take $\bigcirc(\varphi)=\triangle(\varphi \vee \neg \varphi)$ and an obvious computation proves that the operator $\bigcirc$ satisfies the required conditions. 


\section{Conclusions}

In this paper we have been concerned with exploring paraconsistent properties of different kinds of formal systems of fuzzy logic. It has been shown that, while truth-preserving fuzzy logics are not paraconsistent, a class of degree-preserving fuzzy logics are indeed paraconsistent, and some of them can be even considered as proper LFIs, so the fuzzy logic paradigm provides brand new examples of well-behaved paraconsistent logics. As a final remark, we would like to point out that the kind of inconsistencies these paraconsistent fuzzy logics can deal with only arise from the very reason of dealing with intermediate degrees of truth, that is, all these systems immediately become explosive as soon as one forces propositions to be two-valued. Practical inconsistency handling mechanisms using these paraconsistent fuzzy logics remain to be explored.

Acknowledgments All the authors have been partially supported by the FP7 PIRSES-GA-2009-247584 project MaToMUVI. Besides, Ertola was supported by FAPESP LOGCONS Project, Esteva and Godo were supported by the Spanish project TIN2012-39348-C0201, Flaminio was supported by the Italian project FIRB 2010 (RBFR10DGUA_002) and Noguera was supported by the grant P202/10/1826 of the Czech Science Foundation.

\section{References}

[1] Lotfi A. Zadeh. Fuzzy sets. Information and Control, 8(3):338-353, 1965.

[2] Petr Cintula, Petr Hájek, and Carles Noguera, editors. Handbook of Mathematical Fuzzy Logic, Vols. 37 and 38 of Studies in Logic, Mathematical Logic and Foundations. College Publications, London, 2011.

[3] Josep Maria Font, Angel Gil, Antoni Torrens, and Ventura Verdú. On the infinitevalued Łukasiewicz logic that preserves degrees of truth. Archive for Mathematical Logic, 45(7):839-868, 2006.

[4] Félix Bou, Francesc Esteva, Josep Maria Font, Àngel Gil, Lluís Godo, Antoni Torrens, and Ventura Verdú. Logics preserving degrees of truth from varieties of residuated lattices. Journal of Logic and Computation, 19(6):10311069, 2009.

[5] Josep Maria Font. Taking degrees of truth seriously. Studia Logica, 91(3):383-406, 2009.

[6] Philippe Besnard and Anthony Hunter, editors. Reasoning with Actual and Potential Contradictions, Handbook of Defeasible Reasoning and Uncertainty Management Systems, Vol. 2, Kluwer, 1998.

[7] Graham Priest. Paraconsistent logic. In D. Gabbay and F. Guenthner, editors, Handbook of Philosophical Logic, 2nd edition, volume 6, pages 287-393. Kluwer, 2002.
[8] Walter Carnielli, Marcelo E. Coniglio, and João Marcos. Logics of formal inconsistency. In D. Gabbay and F. Guenthner, editors, Handbook of Philosophical Logic, 2nd edition, volume 14, pages 1-93. Springer, 2007.

[9] Ingebrigt Johansson. Der minimalkalkül, ein reduzierter intuitionistischer formalismus. Compositio Mathematica, 4(1):119-136, 1936.

[10] Francesc Esteva and Lluís Godo. Monoidal t-norm based logic: Towards a logic for leftcontinuous t-norms. Fuzzy Sets and Systems, 124(3):271-288, 2001.

[11] Sándor Jenei and Franco Montagna. A proof of standard completeness for Esteva and Godo's logic MTL. Studia Logica, 70(2):183-192, 2002.

[12] Willem J. Blok and Don L. Pigozzi. Algebraizable Logics, volume 396 of Memoirs of the American Mathematical Society, 1989.

[13] Helena Rasiowa. An Algebraic Approach to Non-Classical Logics. North-Holland, Amsterdam, 1974.

[14] Petr Cintula and Carles Noguera. A general framework for mathematical fuzzy logic. In Petr Cintula, Petr Hájek, and Carles Noguera, editors, Handbook of Mathematical Fuzzy Logic - Volume 1, volume 37 of Studies in Logic, Mathematical Logic and Foundations. 103-207, College Publications, 2011.

[15] Carles Noguera. Algebraic Study of Axiomatic Extensions of Triangular Norm Based Fuzzy Logics, volume 27 of Monografies de l'Institut d'Investigació en Intel-ligència Artificial. CSIC, Barcelona, 2007.

[16] Roberto Cignoli, Itala M.L. D'Ottaviano, and Daniele Mundici. Algebraic Foundations of Many-Valued Reasoning, volume 7 of Trends in Logic. Kluwer, Dordrecht, 1999.

[17] Rostislav Horčík, Carles Noguera, and Milan Petrík. On $n$-contractive fuzzy logics. Mathematical Logic Quarterly, 53(3):268-288, 2007.

[18] Cecylia Rauszer. Semi-boolean algebras and their application to intuitionistic logic with dual operators. Fundamenta Mathematicae, 85:219-249, 1974.

[19] Graham Priest. Dualising intuitionistic negation. Principia, 13(2):165-184, 2009.

[20] Rodolfo Ertola. On some operations using the min operator. In Walter Carnielli, Marcelo E. Coniglio, and Itala M.L. D'Ottaviano, editors, The Many Sides of Logic, Vol. 21 of Studies in Logic, pp. 353-368. College Publications, 2009.

[21] José Luis Castiglioni and Rodolfo C. Ertola Biraben. Strict paraconsistency of truth-degree preserving intuitionistic logic with dual negation. Logic Journal of the IGPL, to appear.

[22] Tommaso Flaminio and Enrico Marchioni. Tnorm based logics with an independent involutive negation. Fuzzy Sets and Systems, 157(4):3125-3144, 2006. 